\title{
Bioactive (co)oligoesters with antioxidant properties - synthesis and structural characterization at the molecular level
}

\author{
Magdalena Maksymiak ${ }^{1}$, Tomasz Bałakier ${ }^{2}$, Janusz Jurczak ${ }^{2}$, Marek Kowalczuk $^{1,3}$ and Grazyna \\ Adamus $^{1 *}$ \\ ${ }^{1}$ Polish Academy of Sciences, Centre of Polymer and Carbon Materials, 41-819 Zabrze, Poland \\ ${ }^{2}$ Institute of Organic Chemistry, Polish Academy of Sciences, 01-224 Warsaw, Poland \\ ${ }^{3}$ School of Biology, Chemistry and Forensic Science, Faculty of Science and Engineering, \\ University of Wolverhampton, Wolverhampton WV1 1SB, UK
}

\begin{abstract}
A contemporary synthetic route, starting from the bioactive compound via the corresponding glycidyl ester and $\beta$-substituted $\beta$-lactone to (homo)- and (co)oligoesters with a bioactive moiety covalently linked as pendent groups along an oligomer backbone, was reported. The bioactive compounds were selected from antioxidants used in cosmetics. Two models of bioactive (homo)- and (co)oligoesters were synthesized via anionic ring-opening (co)oligomerization of p-methoxybenzoyloxymethylpropiolactone (p-AA- $\mathrm{CH}_{2}-\mathrm{PL}$ ) initiated by p-anisic acid sodium salt. An analytical protocol was developed for a detailed structural characterization at the molecular level of these bioactive (co)oligoesters. The molecular level structure of the obtained bioactive (homo)- and (co)oligoesters was established by electrospray ionization tandem mass spectrometry (ESI-MS/MS) supported by ${ }^{1} \mathrm{H}$ NMR analysis. Additionally, the results presented here are important for the analysis of designed biodegradable polymeric controlled-release systems of bioactive compounds with potential applications in cosmetology.
\end{abstract}


Keywords: polyhydroxyalkanoates, $\beta$-substituted $\beta$-lactones, $\mathrm{p}$-anisic acid, bioactive (co)oligoesters, anionic ring-opening polymerization

*Corresponding author: Tel.: +48 322716077 /ext. 226/

E-mail address: gadamus@cmpw-pan.edu.pl (G. Adamus)

\section{Introduction}

Increased interest in bioactive compounds is related to the potential possibilities of using them as agents added to food, cosmetics or pharmaceuticals. The term 'bioactive substances' encompasses all components comprising therapeutic and nutritional substances that are normally found in plants in small quantities ${ }^{1}$. A well-known group of bioactive agents are phenolic compounds which include, e.g. flavonoids, phenolic acids or tannins. Phenolic acids are aromatic secondary plant metabolites which are widely spread throughout the plant kingdom. This group include hydroxyl derivatives of cinnamic acid and benzoic acid. Phenolic acids can be easily absorbed in the human system and offer a host of anti-aging benefits. They also reveal very good preservative properties, including antibacterial and antifungal effects ${ }^{2}$.

p-Anisic acid (benzoic acid derivative) possesses antioxidant, anti-tumor and antiinflammatory properties; it has also been found that it has biochemical properties, thus making it suitable as a cosmetic ingredient ${ }^{3}$. p-Anisic acid has recently become increasingly significant as a multi-functional raw material in both the cosmetics and food industries ${ }^{4}$. Today, several antimicrobial fragrance ingredients are commercially available, such as, p-anisic acid (pmethoxybenzoic acid) and levulinic acid (4-oxopentanoic acid), which were found to be the main compounds in Pimpinella anisum and other herbs and in Dioscorea villosa as a by-product in the production of diosgenin from wild yam, respectively ${ }^{5}$. 
The development of macromolecules with a defined structure and properties, aimed specifically for biomedical applications, has resulted in diverse biodegradable polymers with advanced architectures. Recently, the attachment of cosmetics to specific polymer carriers has spurred particular interest. PHAs, mostly poly(3-hydroxybutyrate) and poly(3-hydroxybutyrateco-3-hydroxyvalerate), have been used as carriers for drug delivery because of their biocompatibility, biodegradability and controllable retarding properties which can be modulated by variations in the processing and molecular weight of the polymer composition ${ }^{6-7}$. The synthetic analogue of PHAs can be obtained via anionic ring-opening polymerization of $\beta$ substituted $\beta$-lactones, e.g. atactic poly(3-hydroxybutyrate) is obtained via anionic ROP of $\beta$ butyrolactone ${ }^{8}$.

$\beta$-Lactones are attractive intermediates in organic synthesis ${ }^{9}$ and are useful as monomers in ROP ${ }^{10-12}$. Among the synthetic methods for their preparation, carbonylation of epoxides seems to be the most effective approach. In recent years, highly active and selective catalysts for epoxide carbonylation have been discovered ${ }^{13-15}$, which opened up access to a new source of biologically active $\beta$-lactone monomers and turned out to have very beneficial and far-reaching consequences in the synthesis of new bioactive polyesters, including polymeric delivery systems.

Herein, we report the synthetic strategy for bioactive (co)oligoesters based on anionic homoand co-polymerization of $\beta$-substituted $\beta$-lactones containing bioactive moieties selected from compounds used as preservatives and antioxidants in cosmetics. The ability of these lactones to undergo anionic ring-opening oligomerization in the presence of metal-free anionic initiators has been demonstrated. 
These novel bioactive (co)oligoesters have a larger loading of biologically active substances (p-anisic acid, p-AA) per polymer macromolecule in comparison to the already reported conjugates of oligo(3-hydroxybutyrate) (OHB) with several selected antioxidants ${ }^{16-18}$.

Taking into consideration the prospective use of synthesized new control delivery systems in cosmetics and their contact with the skin, it was important to elucidate the structure and homogeneity of the obtained products.

Previously, mass spectrometry was successfully applied for structural characterization at the molecular level of homopolyesters, copolyesters and their degradation products ${ }^{19-29}$. In our recent works we reported the application of ESI-MS/MS techniques for characterization at the molecular level of OHB conjugates with bioactive compounds including antioxidants used in cosmetics. The molecular structure of bioconjugates, in which antioxidants ( $\alpha$-lipoic, p-coumaric, p-anisic and vanillic acids) are covalently bonded to OHB chains as the end group, has also been proven by tandem mass spectrometry ${ }^{16-18}$. We have also demonstrated the utility of this technique for the analysis of individual molecules of homo- and co-polyesters obtained by anionic ring-opening copolymerization of selected $\beta$-substituted $\beta$-lactones ${ }^{11-12}$.

In the present study, electrospray mass spectrometry was applied in order to obtain more detailed structural information about bioactive (co)oligoesters containing bioactive moieties (panisic acid) along the oligomer chains. The structure of individual macromolecules of the resulting (co)oligoesters (including the chemical structure of their end groups) was determined using ESI-MS ${ }^{\mathrm{n}}$ supported by ${ }^{1} \mathrm{H}$ NMR spectroscopy. The chemical structure of the individual (co)oligoester chains was confirmed by ESI-MS/MS and by investigating the fragmentation product patterns of individual molecular ions.

\section{Experimental Part}


2.1. Materials. $(R, S)-\beta$-Butyrolactone (Sigma-Aldrich Chemie GmbH, Steinheim, Germany) was distilled over $\mathrm{CaH}_{2}$ and the fraction boiling at $56{ }^{\circ} \mathrm{C}$ (9 mmHg) was collected. 4Methoxybenzoyloxymethythylpropiolactone (p-AA-CH$-\mathrm{PL}$ ) was synthesized by carbonylation

of the respective epoxide under ambient CO pressure ${ }^{30} \cdot$ p-Anisic acid sodium salt (sodium panisate, 4-methoxybenzoic acid sodium salt for synthesis, MERCK), dimethylsulfoxide (DMSO, 99.8\%, Aldrich) were used as received.

\subsection{Synthesis of 4-methoxybenzoyloxymethylpropiolactone}

\subsubsection{General considerations}

NMR spectra were recorded using a Varian Mercury spectrometer $\left({ }^{1} \mathrm{H}\right.$ NMR, $500 \mathrm{MHz} ;{ }^{13} \mathrm{C}$ NMR, $125 \mathrm{MHz}$ ) and referenced against tetramethylsilane as an internal standard. The following abbreviations were used to indicate multiplicity: s, singlet; d, doublet; t, triplet; br, broad spin system; m, multiplet; coupling constants are reported in Hz. Analytical TLC was carried out on commercial plates coated with $0.25 \mathrm{~mm}$ Merck Kieselgel 60. Preparative flash silica chromatography was performed using Merck Kieselgel 60 (230-400 mesh). All manipulations of air- and/or water-sensitive compounds were carried out using standard Schlenk line techniques under an atmosphere of dry argon. All carbonylation reactions were set up and run in a wellventilated fume hood equipped with a carbon monoxide detector (see MSDS for proper handling of CO). Dimethoxyethane and methylene chloride were dried by distillation over $\mathrm{CaH}_{2}$ prior to use.

\subsubsection{Preparation of glycidyl 4-methoxybenzoate (p-anisate)}

A catalytic amount of DMF was added (ca. $0.2 \mathrm{~mL}$ ) to a stirred suspension of 4methoxybenzoic acid (11.4 g, $0.075 \mathrm{~mol})$ in $100 \mathrm{~mL}$ of anhydrous methylene chloride. Oxalyl chloride $(7.7 \mathrm{~mL}, 0.09 \mathrm{~mol})$ was then added dropwise and the suspension was stirred at RT until 
it became clear (2-3 h). The solution was then concentrated in vacuo and acid chloride was used directly in the next step without any further purification.

4-Methoxybenzoyl chloride was added dropwise to a stirred solution of glycidol (6mL, 0.09 mol) and triethylamine (12.5 mL, $0.09 \mathrm{~mol})$ in $100 \mathrm{~mL}$ of anhydrous methylene chloride at $0{ }^{\circ} \mathrm{C}$. The reaction mixture was then allowed to reach RT, was stirred for $3 \mathrm{~h}$ and then quenched with saturated $\mathrm{NH}_{4} \mathrm{Cl}$ solution. The organic layer was washed with $1 \mathrm{M} \mathrm{HCl}$, water, brine, dried over anhydrous $\mathrm{MgSO}_{4}$ and concentrated in vacuo. The crude ester was crystallized (ethyl acetate/hexane) and isolated as white crystals (13.5 g, 80\%).

\subsubsection{Procedure for carbonylation of glycidyl 4-methoxybenzoate (p-anisate)}

[N,N’-Bis(3,5-di-tert-butylsalicydene)-1,2-phenylenediaminochromium]chloride (0.9 mmol, 564 $\mathrm{mg}), \mathrm{Co}_{2}(\mathrm{CO})_{8}(1,35 \mathrm{mmol}, 462 \mathrm{mg})$ and glycidyl 4-methoxybenzoate (30 mmol, $\left.6.78 \mathrm{~g}\right)$ were placed in an oven-dried Schlenk tube equipped with a magnetic stir bar under an argon atmosphere. The reaction vessel was then equipped with a latex balloon containing carbon monoxide and was subjected to three vacuum/CO cycles. Anhydrous dimethoxyethane (30 mL) was added via syringe and the reaction was stirred overnight. Then CO gas was carefully vented off. The reaction mixture was evaporated under reduced pressure and the residue was purified by flash column chromatography (hexane, ethyl acetate) to give 4-methoxybenzoyloxymethylpropiolactone (4.95 g, 65\%).<smiles>COc1ccc(C(=O)OCC2CC(=O)O2)cc1</smiles>

${ }^{1} \mathrm{H}$ NMR $\left(\mathrm{CDCl}_{3}, 500 \mathrm{MHz}\right) \delta 7.99$ (d, 2H, J = $\left.9 \mathrm{~Hz}\right), 6.93(\mathrm{~d}, 2 \mathrm{H}, \mathrm{J}=8.8 \mathrm{~Hz}), 4.86(\mathrm{~m}, 1 \mathrm{H})$, 4.69 (dd, $1 \mathrm{H},{ }^{1} \mathrm{~J}=13 \mathrm{~Hz},{ }^{2} \mathrm{~J}=3 \mathrm{~Hz}$ ), 4.53 (dd, 1H, ${ }^{1} \mathrm{~J}=13 \mathrm{~Hz},{ }^{2} \mathrm{~J}=5 \mathrm{~Hz}$ ), 3.87 (s, 3H), 3.62 (dd, 
$\left.1 \mathrm{H},{ }^{1} \mathrm{~J}=16 \mathrm{~Hz},{ }^{2} \mathrm{~J}=6 \mathrm{~Hz}\right), 3.43\left(\mathrm{dd}, 1 \mathrm{H},{ }^{1} \mathrm{~J}=16 \mathrm{~Hz},{ }^{2} \mathrm{~J}=4 \mathrm{~Hz}\right),{ }^{13} \mathrm{C}$ NMR $(125 \mathrm{MHz}) \delta 40.2$, 55.4, 63.4, 67.9, 113.8, 121.3, 131.8, 163.8, 165.6, 166.7; IR (neat, $\mathrm{KBr}$ ) $v_{\mathrm{CO}}=1830 \mathrm{~cm}^{-1}$

2.3. Oligomerization of 4-methoxybenzoyloxymethylpropiolactone in the presence of p-anisic acid sodium salt.

The bioactive (homo)oligoester containing the p-anisic acid moiety e.g. oligo(3-hydroxy-3-(4methoxybenzoyloxymethyl)propionate) (p-AA- $\left.\mathrm{CH}_{2}-\mathrm{HP}\right)_{\mathrm{n}}$ was prepared via anionic ring-opening polymerization of $\beta$-substituted $\beta$-lactone containing the p-anisic acid moiety, e.g. 4methoxybenzoyloxymethylpropiolactone (p-AA- $\mathrm{CH}_{2}-\mathrm{PL}$ ). The reaction was conducted in dimethylosulfoxide (DMSO) at room temperature in a glass reactor that was flamed and dry argon-purged prior to use. The monomer p-AA- $\mathrm{CH}_{2}-\mathrm{PL}(0.20 \mathrm{~g}, 0.85 \mathrm{mmol})$ was added to reactors that contained the solvent DMSO $(4 \mathrm{~mL})$ and the required amount of initiator: p-anisic acid sodium salt (sodium p-anisate, $\left.\left(\mathrm{CH}_{3} \mathrm{OPhC}(\mathrm{O}) \mathrm{O}^{-} \mathrm{Na}^{+}\right), 0,02 \mathrm{~g}, 0,13 \mathrm{mmol}\right)$. The average molar mass of the conjugates was controlled by the monomer-initiator ratio. The progress of polymerization was monitored by Fourier transform infrared spectroscopy (FT-IR) based on the intensity of the signals arising from the carbonyl groups of the monomer and oligomer at 1837 and $1743 \mathrm{~cm}^{-1}$, respectively. After the completion of oligomerization, the resulting oligomers were precipitated from cold hexane and dried under vacuum. Chloroform solutions of oligomers were acidified with dilute $\mathrm{HCl}(\mathrm{aq})$ and the mixtures were stirred vigorously for $10 \mathrm{~min}$. After phase separation, the organic layer was removed and acidified again (as described above). Then the oligomer solution was washed 10 times with $5 \mathrm{~mL}$ of distilled water. Next, the solvent was evaporated and the oligomers were dried under vacuum at room temperature for $48 \mathrm{~h}$ and analyzed. 
2.4. (Co)oligomerization of 4-methoxybenzoyloxymethylpropiolactone in the presence of p-anisic acid sodium salt.

The bioactive random (co)oligoester containing the p-anisic acid moiety e.g. oligo[(3-hydroxy3-(4-methoxybenzoyloxymethyl)propionate)-co-(3-hydroxybutyrate)] was prepared via anionic ring-opening copolymerization of $\beta$-substituted $\beta$-lactone containing the p-anisic moiety (p-AA$\mathrm{CH}_{2}-\mathrm{PL}$ ) with $\beta$-butyrolactone ( $\beta$-BL). The reaction was performed under an argon atmosphere at room temperature. Monomers p-AA-CH $2-\mathrm{PL}(0.20 \mathrm{~g}, 0.85 \mathrm{mmol})$ and $\beta$-BL $(0.073 \mathrm{~mL}, 0.85$ mmol) were added to reactors containing the solvent: DMSO $5 \mathrm{~mL}$ and the required amount of initiator: sodium p-anisate, $\left(\mathrm{CH}_{3} \mathrm{OPhC}(\mathrm{O}) \mathrm{O}^{-} \mathrm{Na}^{+}\right),(0.031 \mathrm{~g}, 0.18 \mathrm{mmol})$. Progress of the copolymerization was monitored by FT-IR spectroscopy based on the intensity of the carbonylgroup signals of the monomers at $1837 \mathrm{~cm}^{-1}\left(\mathrm{p}-\mathrm{AA}-\mathrm{CH}_{2}-\mathrm{PL}\right)$ and $1830 \mathrm{~cm}^{-1}(\beta-\mathrm{BL})$ and that of the oligomers at $1740 \mathrm{~cm}^{-1}$. The resulting (co)oligoesters were purified as described above.

\subsection{Measurements:}

\subsubsection{Size-Exclusion Chromatography (SEC) analysis}

Number-average and weight-average molar mass and molar mass distribution $\left(\mathrm{M}_{\mathrm{w}} / \mathrm{M}_{\mathrm{n}}\right)$ were determined by SEC conducted in chloroform at $35{ }^{\circ} \mathrm{C}$ with a flow rate of $1 \mathrm{~mL} \mathrm{~min}{ }^{-1}$ using an isocratic pump (VE 1122, Viscotek), a PLgel 3 um MIXED-E (Polymer Laboratories) ultra-high efficiency column (300 mm × $7.5 \mathrm{~mm}$ ) and a Shodex SE 61 differential refractive index detector. The injection volume was $100 \mu \mathrm{L}$ of the sample in chloroform $(0.3 \% \mathrm{w} / \mathrm{v})$. Polystyrene standards (Polymer Laboratories) with narrow molar mass distributions were used to generate a calibration curve.

2.5.2. Nuclear magnetic resonance (NMR) analysis: 
${ }^{1} \mathrm{H}$ NMR spectra of the homo- and copolyesters were recorded using a Bruker-Avance 600 $\mathrm{MHz}$ multinuclear magnetic resonance spectrometer. The analyses were run in $\mathrm{CDCl}_{3}$ using tetramethylsilane (TMS) as an internal standard.

\subsubsection{Fourier transform infrared spectroscopy (FTIR) analysis:}

FTIR spectra were recorded using a FTS 40A Bio-Rad spectrometer at room temperature.

\subsubsection{Electrospray mass spectrometry $\left(E S I-M S^{n}\right)$ analysis:}

Electrospray mass spectrometry analysis was performed using a Finnigan LCQ ion trap mass spectrometer (Finnigan, San Jose, CA, USA). The obtained bioactive (co)oligoesters were dissolved in methanol (99.8\%, pure p.a.-basic, POCH SA). The solutions were introduced into the ESI source by continuous infusion at a rate of $10 \mu \mathrm{L} \mathrm{min}{ }^{-1}$ using an instrument syringe pump. The LCQ ESI source was operated at $4.5 \mathrm{kV}$ and the capillary was heated to $200{ }^{\circ} \mathrm{C}$. Nitrogen was used as the nebulizing gas. For the ESI-MS/MS experiments, monoisotopic ions of interest were isolated in the ion trap and collisionally activated using the helium damping gas present in the mass analyzer as a collision gas. The RF amplitude, which had a substantial voltage range, was set such that the peak height of the molecular ion decreased by at least $50 \%$. The analyses were performed in positive-ion mode.

\section{Results and discussion}

In recent years, much attention has been focused on the synthesis of $\beta$-lactones by catalytic carbonylation of epoxides ${ }^{13-15}$. This is a relatively simple synthetic method, thus the obtained monomers may be used on a large scale for the synthesis of new biodegradable homo- and copolymers with specific characteristics, e.g. for prospective applications of controlled release systems of biologically active substances ${ }^{30}$. The monomer, 4-methoxybenzoyloxymethylpropiolactone (p-AA- $\mathrm{CH}_{2}-\mathrm{PL}$ ), which was used in this study was prepared by 
carbonylation of a suitable glycidyl ester (Scheme 1a). The resulting new $\beta$-substituted $\beta$-lactone containing covalently attached p-anisic acid was used for the synthesis of biologically active (homo)- and (co)oligoesters for prospective applications in cosmetology in controlled release systems. Scheme $1 \mathrm{~b}$ describes the anionic ring-opening oligomerization of $\mathrm{p}-\mathrm{AA}-\mathrm{CH}_{2}-\mathrm{PL}$ initiated by sodium p-anisate which led to the bioactive (homo)oligoester: oligo(3-hydroxy-3-(4methoxybenzoyloxymethyl)propionate), (p-AA- $\left.\mathrm{CH}_{2}-\mathrm{HP}\right)_{\mathrm{n}}$ (Sample 1, Table 1). Whereas the bioactive (co)oligoester: oligo[(3-hydroxy-3-(4-methoxybenzoyloxymethyl)propionate)-co-(3hydroxybutyrate)] (p-AA-CH2-HP) m $\mathrm{HB}_{\mathrm{n}}$ (Sample 2, Table 1) was synthesized via anionic ringopening (co)oligomerization of p-AA- $\mathrm{CH}_{2}$-PL with $\beta$-butyrolactone ( $\beta$-BL) initiated by sodium p-anisate (Scheme 1c).

\section{Scheme 1}

The obtained bioactive (homo)- and (co)oligoesters were preliminarily characterized by SEC,

${ }^{1} \mathrm{H}$ NMR techniques and ESI-MS ${ }^{\mathrm{n}}$. The results of this characterization of the obtained products by SEC are summarized in Table 1.

Table 1 (Homo)- and (co)oligoester characteristics by SEC.

\begin{tabular}{|c|c|c|c|}
\hline Sample & $\begin{array}{c}\text { Composition [mol \% }]^{\mathbf{a}} \\
\left.\mathbf{( p - A A - C H}_{\mathbf{2}} \mathbf{H P}\right) / \mathbf{H B}\end{array}$ & $\begin{array}{c}\mathbf{M}_{\mathbf{n}}{ }^{\mathbf{b}} \\
{[\mathbf{g} / \mathbf{m o l}]}\end{array}$ & $\mathbf{M}_{\mathbf{w}} / \mathbf{M}_{\mathbf{n}}{ }^{\mathbf{c}}$ \\
\hline $\mathbf{1}$ & $100 / 0$ & 700 & 1.15 \\
\hline $\mathbf{2}$ & $50 / 50$ & 900 & 1.23 \\
\hline
\end{tabular}

${ }^{\text {a) }}$ Molar composition of (co)oligoesters estimated from ${ }^{1} \mathrm{H}$ NMR.

b) Number-average molar mass and ${ }^{\mathrm{c})}$ molar mass distribution estimated by SEC.

The chemical structures of the bioactive (homo)- and (co)oligoesters obtained in this study were determined by ${ }^{1} \mathrm{H}$ NMR spectroscopy. The ${ }^{1} \mathrm{H}$ NMR spectrum of (p-AA-CH$\left.-\mathrm{HP}\right)_{\mathrm{m}} / \mathrm{HB}_{\mathrm{n}}$ is 
presented in Figure 1. The ${ }^{1} \mathrm{H}$ NMR spectrum reveals signals ascribed to the protons of the methyl, methylene and methine groups of 3-hydroxybutyrate and 3-hydroxy-3-(4methoxybenzoyloxymethyl)propionate repeating units (corresponding signals: 3, 3', 1 and 1' as well as $\mathbf{2}$ and 2'). Additionally, overlapping signals corresponding to the protons of the terminal group (derived from the incorporated initiator) and p-anisic acid embedded as side groups along the p-AA- $\mathrm{CH}_{2}-\mathrm{HP}$ chain (signals 4-6', Figure 1) were detected in the spectrum.

\section{Figure 1}

3.1. Molecular structural studies of the (homo)oligoester containing bioactive p-anisic acid moieties

Electrospray ion trap tandem mass spectrometry (ESI-MS ${ }^{\mathrm{n}}$ ) was applied to obtain detailed information about the structure of the resulting $\left(\mathrm{p}-\mathrm{AA}-\mathrm{CH}_{2}-\mathrm{HP}\right)_{\mathrm{n}}$ oligoester at the molecular level.

The ESI mass spectrum of $\left(\mathrm{p}-\mathrm{AA}-\mathrm{CH}_{2}-\mathrm{HP}\right)_{\mathrm{n}}$ oligomers obtained here is depicted in Figure 2. One main set of ions with a peak-to-peak mass increment of 236 Da was observed in the mass spectrum. The structures of the end groups and repeating units can be inferred based on the mass assignment of singly charged ions observed in the mass spectrum. The series of ions labeled as $\left(\mathrm{p}-\mathrm{AA}-\mathrm{CH}_{2}-\mathrm{HP}\right)_{1}-\left(\mathrm{p}-\mathrm{AA}-\mathrm{CH}_{2}-\mathrm{HP}\right)_{7}$ correspond to the sodium cationized 3hydroxy-3-(4-methoxybenzoyloxymethyl)propionate oligomers terminated by pmethoxybenzoyl (p-anisate, derived from the initiator) and carboxyl end groups.

\section{Figure 2}

Tandem mass spectrometry (ESI-MS/MS) was used for further structural elucidation of the oligomers' structure. In this technique, the molecular ion of interest is separated from the other ions formed during the electrospray ionisation process and is induced to dissociate into 
fragments. These can be used for structural characterization (including end-group analysis) of the individual polymer chains. To verify the structure of individual ions belonging to the main series, ESI-MS/MS fragmentation experiments were performed for ions selected from this series.

Previously published studies on the fragmentation of individual precursor ions of polyesters (derivatives of $\beta$-hydroxy acids) revealed that $\beta$-hydrogen rearrangement was the main mechanism inducing fragmentation of such polyesters via ester bond cleavage ${ }^{26,32-34}$.

Figure 3 shows the ESI-MS/MS spectrum of the ion at $\mathrm{m} / \mathrm{z} 883$ corresponding to the sodium adduct of the oligoester containing three 3-hydroxy-3-(4-methoxybenzoyloxymethyl)propionate repeating units terminated by p-anisate and carboxyl end groups. The fragmentation pathway of the ion at $\mathrm{m} / \mathrm{z}$ 883, which occurs as a result of random breakage of ester bonds along the oligomer backbone and of ester bonds between the backbone and the bioactive pendant groups, is shown in Figure 3. The product ion at $m / z 731$ corresponds to the oligomer formed by the loss of p-anisic acid (152 Da) derivative from the oligomer terminal group or from the bioactive pendant group of the p-AA- $\mathrm{CH}_{2}-\mathrm{HP}$ chain; whereas the product ion at $\mathrm{m} / \mathrm{z} 647$ corresponds to the oligomer formed by the loss of 4-methoxybenzoyloxycrotonic acid (236 Da). A comparison of the fragmentation spectra and the theoretical path of the ion fragmentation showed that all theoretically predicted structures of the product ions are shown at the spectrum, which confirms the structure of the obtained bioactive oligoester (Figure 3).

\section{Figure 3}

\subsection{ESI-MS reveals the random structure of (p-AA-CH$-\mathrm{HP})_{m} / \mathrm{HB}_{n}$ (co)oligoesters}

The ESI-mass spectrum (positive ion-mode) of oligomers obtained by (co)oligomerization of $\beta$-BL with p-AA- $\mathrm{CH}_{2}$-PL initiated by sodium p-anisate (together with spectral expansion in the range $m / z$ 650-1050) are depicted in Figure 4. Three series of singly charged ions (labeled as A, 
B and C) that correspond to the three types of oligomers are present in the mass spectrum of the sample in Figure 4. Two main sets of ions, which appear in the mass spectrum at $m / z=151+(n \times$ $86)+(m \times 236)+1+23$ (Figure 4) with a peak-to-peak mass increment of 236 Da (labeled as Series A and B), were observed in the mass spectrum. The signals belonging to Series A and B correspond to sodium cationized $\left[\left(\mathrm{p}-\mathrm{AA}-\mathrm{CH}_{2}-\mathrm{HP}\right)_{\mathrm{m}} / \mathrm{HB}_{\mathrm{n}}+\mathrm{Na}\right]^{+}$(co)oligoester chains terminated by p-anisic acid and carboxylic end groups and containing one (Series A) or two (Series B) (p-AA- $\mathrm{CH}_{2}-\mathrm{HP}$ ) comonomer units that can be arranged with different combinations along the oligomer backbone. The third and less abundant series of ions (with a lower relative intensity) labeled Series C (see Figure 4) was observed in the mass spectra at $\mathrm{m} / \mathrm{z}=151+(n x$ 86) $+1+23$. These series can be assigned to sodium cationized oligo(3-hydroxybutyrate) terminated by p-anisate and carboxyl end groups. Thus the mass spectrometry analysis indicated that the (co)oligoester contained one, two or three bioactive p-anisic moieties distributed along the oligomer chains was synthesized (Figure 4). The general structures of the ions present in the ESI-MS spectra (Figure 4) are depicted in Table 2.

The presence of these oligomer chains can be expected at each degree of polymerization, and their amount depends on the comonomer ratio, polymerization mechanism, degree of copolymerization (e.g. the number of comonomeric units in a macromolecule) as well as on the comonomer composition distribution.

\section{Figure 4}


Table 2 Structural assignments of ions appearing in the expanded region $(\mathrm{m} / \mathrm{z}$ 660-1020) of the ESI-MS spectrum of oligomers obtained by (co)oligomerization of $\beta$-BL with p-AA-CH$-\mathrm{PL}$ initiated by sodium p-anisate.

\begin{tabular}{|c|c|c|c|}
\hline \multirow[b]{2}{*}{ Series } & \multirow[b]{2}{*}{ Chemical Structure } & Composition & \multirow[b]{2}{*}{$m / z$} \\
\hline & & (p-AA-CH $2-H P) / H B$ & \\
\hline $\mathbf{A}$ & & $\begin{array}{l}\left(\mathrm{p}-\mathrm{AA}-\mathrm{CH}_{2}-\mathrm{HP}\right)_{1} / \mathrm{HB}_{3} \\
\left(\mathrm{p}-\mathrm{AA}-\mathrm{CH}_{2}-\mathrm{HP}\right)_{1} / \mathrm{HB}_{4} \\
(\text { p-AA-CH } \\
\left.\text { (p-AA-CH }{ }_{2}-\mathrm{HP}\right)_{2}-\mathrm{HB}_{5} \\
\left(\mathrm{H}-\mathrm{AA}-\mathrm{CB}_{2}-\mathrm{HP}\right)_{1} / \mathrm{HB}_{7}\end{array}$ & $\begin{array}{c}669 \\
755 \\
841 \\
927 \\
1013\end{array}$ \\
\hline B & & $\begin{array}{l}\left(\mathrm{p}-\mathrm{AA}-\mathrm{CH}_{2}-\mathrm{HP}\right)_{2} / \mathrm{HB}_{1} \\
\left(\mathrm{p}-\mathrm{AA}-\mathrm{CH}_{2}-\mathrm{HP}\right)_{2} / \mathrm{HB}_{2} \\
\left(\mathrm{p}-\mathrm{AA}-\mathrm{CH}_{2}-\mathrm{HP}\right)_{2} / \mathrm{HB}_{3} \\
\left(\mathrm{p}-\mathrm{AA}-\mathrm{CH}_{2}-\mathrm{HP}\right)_{2} / \mathrm{HB}_{4}\end{array}$ & $\begin{array}{l}733 \\
819 \\
905 \\
991\end{array}$ \\
\hline C & & $\begin{array}{l}\mathrm{HB}_{6} \\
\mathrm{HB}_{7} \\
\mathrm{HB}_{8} \\
\mathrm{HB}_{9}\end{array}$ & $\begin{array}{l}691 \\
777 \\
863 \\
949\end{array}$ \\
\hline
\end{tabular}


3.3. Structural characterization of random ( $\left.p-A A-C H_{2}-H P\right)_{m} / H B_{n}$ (co)oligoesters with tandem ESI-MS/MS

More detailed insight into the structure of individual (co)oligoester chains may be obtained by using tandem ESI-MS/MS spectrometry. This technique has been applied for structural studies and investigation of fragmentation product patterns of individual molecular ions while allowing differentiation between individual molecular chains of random and diblock copolyesters ${ }^{33}$. Figure 5 presents the ESI-MS/MS spectrum (in positive-ion mode) of the precursor ion at $\mathrm{m} / \mathrm{z}$ 841, which corresponds to the sodium adduct of oligomers terminated by carboxyl and panisate (derived from the initiator used) end groups and containing five 3-hydroxybutyrate repeating units and one p-AA- $\mathrm{CH}_{2}-\mathrm{HP}$ unit in the (co)oligoester chain. Fragmentation of this precursor ion at $m / z 841$ (belonging to Series A, Figure 4) takes place as a result of random breakage of ester bonds along the oligomer chain and ester bonds between the chain and the bioactive pendant group (see the fragmentation pathway in Figure 5 and Scheme 2). Thus the product ion at $m / z 689$ corresponds to the oligomer formed by the loss of p-anisic acid (152 Da), which can be derived from the terminal group and/or from the bioactive pendant group of the pAA- $\mathrm{CH}_{2}-\mathrm{HP}$ comonomer unit. While the product ion at $\mathrm{m} / \mathrm{z} 755$ corresponds to the oligomer formed by the loss of the last 3-hydroxybutyrate unit in the oligoester chains in the form of crotonic acid (86 Da), the formation of the product ion at $\mathrm{m} / \mathrm{z} 605$ is associated with the loss of 4methoxybenzoyloxycrotonic acid (236 Da, see Scheme 2) if the last comonomer unit in the oligoester chain is p-AA- $\mathrm{CH}_{2}-\mathrm{HP}$. All theoretically predicted product ions are present in the ESIMS/MS spectrum in Figure 5, which is in good agreement with the theoretical fragmentation pathways we proposed in Scheme 2. Moreover, such a fragmentation pathway indicates that the p-AA- $\mathrm{CH}_{2}-\mathrm{HP}$ comonomer unit is randomly distributed along the oligomer chain. 
Figure 6 shows the ESI-MS/MS product ions' spectrum of the precursor ion at $\mathrm{m} / \mathrm{z} 819$ corresponding to the sodium adduct of $\left[\mathrm{p}-\mathrm{AA}-\left(\mathrm{p}-\mathrm{AA}-\mathrm{CH}_{2}-\mathrm{HP}\right)_{2} / \mathrm{HB}_{2}+\mathrm{Na}^{+}\right.$] (co)oligoester chains terminated by p-anisate and carboxyl end groups, containing two $\mathrm{HB}$ and two p-AA- $\mathrm{CH}_{2}-$ HP repeating units in the (co)oligoester chain. In this case, random breakage of ester bonds along the oligomer chain and ester bonds between the chain and the bioactive pendant group led to the formation of two series of product ions at $m / z$ 733, 667, 647, 583, 581, 497, 495, 431, 411.

Thus the product ion at $\mathrm{m} / \mathrm{z} 667$ corresponds to the oligomer formed by the loss of the p-anisic acid molecule (152 Da), a derivative from the oligomer end group or from the bioactive pendant group of the p-AA- $\mathrm{CH}_{2}-\mathrm{HP}$ comonomer unit. While the product ion at $\mathrm{m} / \mathrm{z} 733$ corresponds to the oligomer formed by the loss of HB units in the form of crotonic acid if the HB unit is the last comonomer unit in the oligoester chain (86 Da; see Figure 6 and Scheme 3). The product ion at $\mathrm{m} / \mathrm{z} 583$ corresponds to the oligomer formed by the loss of the last, in the oligoester chain, p-AA- $\mathrm{CH}_{2}-\mathrm{HP}$ comonomer unit in the form of 4-methoxybenzoyloxycrotonic acid (236 Da, see Scheme 3). Such a fragmentation pathway indicates that the two p-AA- $\mathrm{CH}_{2}$ HP comonomer units are randomly distributed along the oligomer chain ${ }^{33}$.

ESI-MS/MS experiments were also performed for ions derived from Series C (assigned as $\mathrm{HB}_{\mathrm{n}}$ in the spectral expansion in Figure 4). The selected ions correspond to the sodium adducts of pAA-oligo(3-hydroxybutyrate) (p-AA-OHB) chains terminated by p-anisate and carboxylic end groups. Fragmentation of these ions proceeds via a similar fragmentation pathway and was previously reported in ref. ${ }^{18}$.

\section{Figure 5}

\section{Scheme 2}

\section{Figure 6}




\section{Scheme 3}

\section{Conclusions}

The results of the present study revealed that anionic oligomerization of $\beta$-substituted $\beta$ lactones containing covalently bonded p-anisic acid enabled the formation of bioactive (homo)oligoesters having from 1 to 8 molecules of p-anisic acid and (co)oligoesters containing from 2 to 3 molecules of p-anisic acid bound to the polymer backbone as a bioactive terminal and pendant groups through hydrolyzable ester linkages.

An analytical method for a detailed structural characterization at the molecular level of bioactive (co)oligoesters has been developed. The molecular structures of the obtained (homo)and (co)oligoesters were determined with the use of ESI-mass spectrometry supported by ${ }^{1} \mathrm{H}$ NMR analyses. Structures of the end groups and copolymer composition were verified by ESIMS/MS. It was shown that fragmentation of selected ions of bioactive (co)oligoesters proceeded via random breakage of ester bonds along the oligomer chain as well as of ester bonds of the bioactive pendant group. Additionally, the ESI-MS/MS fragmentation experiments conducted for selected sodium adducts of the obtained (co)oligoesters confirmed that the bioactive moieties were covalently bonded to oligo(3-hydroxybutyrate) chains as terminal and pendant groups. Moreover, the fragmentation pathway of selected oligomers indicated that the p-AA- $\mathrm{CH}_{2}-\mathrm{HP}$ comonomer units were randomly distributed along the (co)oligoester chain. Thus the obtained results demonstrate the utility of this technique for the analysis of individual molecules of the bioactive (homo)- and (co)oligoesters studied here.

Novel delivery systems obtained via the elaborated synthetic strategy contain a larger loading of biologically active substances per polymer macromolecule than the respective 
cosmetic delivery system that we have already reported ${ }^{16-18}$. The object of further work will be to carry out preliminary studies proving the usefulness of the developed (co)oligoesters as new controlled release systems for applications in cosmetology.

\section{Acknowledgments}

This work was supported by the National Science Centre: project titled "Studies on the synthesis of novel biodegradable control release systems of bioactive compounds for perspective applications in cosmetology”, decision no. DEC-2013/09/N/ST5/00890 and project titled “Low pressure catalytic synthesis of novel beta-lactone monomers and their anionic ring-opening (co)polymerization leading to synthetic analogues of aliphatic biopolyesters”, decision no. DEC2012/07/B/ST5/00627.

\section{References}

[1] P.M. Kris-Etherton, K.D. Hecker and A. Bonanome, Bioactive compounds in foods: their role in the prevention of cardiovascular disease and cancer, Am J Med, 2002, 113, 71S.

[2] C.A. Rice-Evans, N.J. Miller and G. Paganga, Structure-antioxidant activity relationships of flavonoids and phenolic acids, Free Radic Biol Med., 1996, 20, 933.

[3] S. Papageorgiou, A. Varvaresou, E. Tsirivas and C. J. Demetzos, New alternatives to cosmetics preservation, Cosmet. Sci., 2010, 61, 107.

[4] U. Straetmans, J. Janichen, W. Petersen, M. Kinder, C. Johnson and G. Reynolds, Concentrated, aqueous solutions of p-methoxybenzoic acid for use in cosmetic and dermatologic formulations. US 20060229291 A1, Oct 12, 2006. 
[5] M. Sautour, A.-C. Mitaine-Offer and M.-A Lacaille-Dubois, The Dioscorea genus: A review of bioactive steroid saponins, J. Nat. Med., 2007, 61, 91.

[6] A. J. Anderson and E. A. Dawes, Occurrence, metabolism, metabolic role, and industrial uses of bacterial polyhydroxyalkanoates, Microbiol. Mol. Biol. Rev. 1990, 54, 450.

[7] A. Shrivastav, H.-Y. Kim and Y.-R. Kim, Advances in the Applications of Polyhydroxyalkanoate Nanoparticles for Novel Drug Delivery System, BioMed Res. Int., 2013, 2013, 1.

[8] Z. Jedliński, M. Kowalczuk, W. Główkowski, J. Grobelny, M. Szwarc, Novel polymerization of $\beta$-butyrolactone initiated by potassium naphthalenide in the presence of a crown ether or a cryptand, Macromolecules, 1991, 24, 349.

[9] A. Pommier and J. M. Pons, Recent Advances in $\beta$-Lactone Chemistry, Synthesis, 1993, 5, 441.

[10] L. R. Rieth, D. R Moore, E. B. Lobkovsky and G. W. Coates, Single-Site ß-Diiminate Zinc Catalysts for the Ring-Opening Polymerization of $\beta$-Butyrolactone and $\beta$ Valerolactone to Poly(3-hydroxyalkanoates), J. Am. Chem. Soc., 2002, 124, 15239.

[11] G. Adamus and M. Kowalczuk, Synthesis of Poly(2-methyl-3-hydroxyoctanoate) via Anionic Polymerization of $\alpha$-Methyl- $\beta$-pentyl- $\beta$-propiolactone, Biomacromolecules, 2008, 9, 696.

[12] G. Adamus, I. Kwiecień, M. Maksymiak, T. Bałakier, J. Jurczak and M. Kowalczuk, Molecular level structure of novel synthetic analogues of aliphatic biopolyesters as revealed by multistage mass spectrometry, Anal. Chim. Acta., 2014, 808, 104. 
[13] Y. D. Y. L. Getzler, V. Mahadevan, E. B. Lobkovsky and G. W. Coates, Synthesis of ßLactones: A Highly Active and Selective Catalyst for Epoxide Carbonylation, J. Am. Chem. Soc., 2002, 124, 1174.

[14] J. A. R. Schmidt, V. Mahadevan, Y. D. Y. L Getzler and G. W. Coates, A Readily Synthesized and Highly Active Epoxide Carbonylation Catalyst Based on a Chromium Porphyrin Framework. Expanding the Range of Available ß-Lactones, Org. Lett., 2004, 6, 373.

[15] J. W. Kramer, E. B. Lobkovsky and G. W. Coates, Practical ß-Lactone Synthesis: Epoxide Carbonylation at 1 atm, Org. Lett. 2006, 8, 3709.

[16] M. Maksymiak, R. Dębowska, K. Jelonek, M. Kowalczuk and G. Adamus, Structural characterization of biocompatible lipoic acid-oligo-(3-hydroxybutyrate) conjugates by electrospray ionization mass spectrometry, Rapid Commun. Mass Spectrom., 2013, 27, 773.

[17] M. Maksymiak, M. Kowalczuk and G. Adamus, Electrospray tandem mass spectrometry for the structural characterization of p-coumaric acid-oligo(3-hydroxybutyrate) conjugates, Int. J. Mass Spectrom., 2014, 359, 6.

[18] M. Maksymiak, R. Debowska, K. Bazela, A. Dzwigalowska, A. Orchel, K. Jelonek, B. Dolegowska, M. Kowalczuk and G Adamus, Designing of Biodegradable and Biocompatible Release and Delivery Systems of Selected Antioxidants Used in Cosmetology, Biomacromolecules, 2015, 16, 3603.

[19] G. Montaudo and R.P. Lattimer, Mass Spectrometry of Polymers, CRC Press, Boca Raton, London, New York, Washington D.C., 2002. 
[20] A. C. Crecelius, A. Baumgaertel and U.S. Schubert, Tandem mass spectrometry of synthetic polymers, J. Mass Spectrom., 2009, 44, 1277.

[21] S. Koster, M.C. Duursma, J. J. Boon, M.W.F. Nielen, C.G. Koster and R.M.A. Heeren, Structural analysis of synthetic homo- and copolyesters by electrospray ionization on a Fourier transform ion cyclotron resonance mass spectrometer, J. Mass Spectrom., 2000, 35, 739.

[22] M.A Arnould, R. Vargas, R.W. Buehner and C. Wesdemiotis, Tandem Mass Spectrometry Characteristics of Polyester Anions and Cations Formed by Electrospray Ionization, Eur. J. of Mass Spectrom., 2005, 11, 243.

[23] J. De Winter, O. Coulembier, P. Dubois and P. Gerbaux, Collision-induced dissociation of polymer ions : charge driven decomposition for sodium-cationized polylactides, Int. J. Mass Spectrom., 2011, 308, 11.

[24] I. Osaka, M. Watanabe, M. Takama, M. Murakami and R. Arakawa, Characterization of linear and cyclic polylactic acids and their solvolysis products by electrospray ionization mass spectrometry, J. Mass Spectrom., 2006, 41, 1369.

[25] T. Gruendling, S. Weidner, J. Falkenhagen and C. Barner-Kowollik, Mass spectrometry in polymer chemistry: a state-of-the-art up-date, Polym. Chem., 2010, 1, 599.

[26] C. Barner-Kowollik, T. Gründling, J. Falkenhagen, S. Weidner (Eds.), Mass Spectrometry in Polymer Chemistry, Viley-VCH, Weinheim, 2011.

[27] T. Josse, J. De Winter, P. Dubois, O. Coulembier, P. Gerbaux and A. Memboeuf, A tandem mass spectrometry-based method to assess the architectural purity of synthetic 
polymers: case of a cyclic polylactide obtained by click chemistry, Polymer Chemistry, 2015, 6, 64.

[28] M. Hakkarainen, G. Adamus, A. Höglund, M. Kowalczuk and A.-C. Albertsson, ESI-MS Reveals the Influence of Hydrophilicity and Architecture on the Water-soluble Degradation Product Patterns of Biodegradable Homo- and Copolyesters of 1,5dioxepan-2-one and ع-caprolactone, Macromolecules, 2008, 41, 3547.

[29] A. Höglund, M. Hakkarainen, M. Kowalczuk, G. Adamus and A.-C. Albertsson, Fingerprinting the Degradation Product Patterns of Different Polyester-ether Networks by Electrospray Ionization Mass Spectrometry, J. Polym. Sci., Part A:Polym. Chem., 2008, 46, 4617.

[30] T. Bałakier, W. Chaładaj, J. Jurczak, G. Adamus and M. Kowalczuk, An effective protocol for the synthesis enantiomerically pure 4-substituted oxetane-2-ones, Tetrahedron, 2013, 69, 4990.

[31] S. M. Hunt, M.R Binns and M. Sheil, Structural characterization of polyester resins by electrospray mass spectrometry, J. Appl Polym. Sci., 1995, 56,1589.

[32] P. Rizzarelli, C. Puglisi, G. Montaudo, Matrix-assisted laser desorption/ionization timeof-flight/time-of-flight tandem mass spectra of poly(butylene adipate), Rapid Commun, Mass Spectrom., 2006, 20, 1683.

[33] G. Adamus, Molecular Level Structure of (R,S)-3-Hydroxybutyrate/(R,S)-3-Hydroxy-4ethoxybutyrate Copolyesters with Dissimilar Architecture, Macromolecules, 2009, 42, 4547. 
[34] E. Zagar, A. Krzan, G. Adamus and M. Kowalczuk, Sequence distribution in microbial poly(3-hydroxybutyrate-co-3-hydroxyvalerate) co-polyesters determined by NMR and MS, Biomacromolecules, 2006, 7, 2210.

\section{Figure captions:}

Figure 1. ${ }^{1} \mathrm{H}$ NMR spectrum (in $\mathrm{CDCl}_{3}$ ) of the (p-AA- $\left.\mathrm{CH}_{2}-\mathrm{HP}\right)_{\mathrm{m}} / \mathrm{HB}_{\mathrm{n}}$ oligomer obtained via cooligomerization of $\beta$-BL with p-AA- $\mathrm{CH}_{2}$-PL initiated by sodium p-anisate.

Figure 2. ESI-mass spectrum (positive-ion mode) of the (p-AA- $\left.\mathrm{CH}_{2}-\mathrm{HP}\right)_{\mathrm{n}}$ (homo)oligoester obtained via anionic ring-opening oligomerization (p-AA- $\mathrm{CH}_{2}-\mathrm{PL}$ ) initiated by sodium p-anisate.

Figure 3. ESI-MS/MS spectrum of the sodium adduct of oligomers containing three 3-hydroxy3-(4-methoxybenzoyloxymethyl)propionate repeating units and the theoretical fragmentation pathway.

Figures 4. ESI-mass spectra (positive ion-mode) of oligomers obtained by (co)oligomerization of $\beta$-BL with p-AA- $\mathrm{CH}_{2}-\mathrm{PL}$ initiated by sodium $\mathrm{p}$-anisate and spectral expansion in the mass range $m / z$ 660-1020.

Figure 5. ESI-MS/MS spectrum (in positive-ion mode) of a selected sodium adduct of [p-AA$\left.\left(\mathrm{p}-\mathrm{AA}-\mathrm{CH}_{2}-\mathrm{HP}\right)_{1} / \mathrm{HB}_{5}+\mathrm{Na}\right]^{+}$(co)oligoester at $\mathrm{m} / \mathrm{z} 841$ terminated by p-anisic acid and carboxylic end groups and containing one p-AA- $\mathrm{CH}_{2}-\mathrm{HP}$ comonomer unit.

Figure 6. ESI-MS/MS spectrum (in positive-ion mode) of a selected sodium adduct of [p-AA-(pAA- $\left.\left.\mathrm{CH}_{2}-\mathrm{HP}\right)_{2} / \mathrm{HB}_{2}+\mathrm{Na}\right]^{+}$(co)oligoester at $m / z 819$ terminated by p-anisic acid and carboxylic end groups and containing two p-AA- $\mathrm{CH}_{2}-\mathrm{HP}$ comonomer units. 
Scheme 1. Synthesis of (a) 4-methoxybenzoyloxymethylpropiolactone, (b) oligo(3-hydroxy-3(4-methoxybenzoyloxymethyl)propionate), and (c) oligo[(3-hydroxy-3-(4-methoxybenzoyloxymethyl)propionate)-co-(3-hydroxybutyrate)].

Scheme 2. Theoretical fragmentation pathway of the sodium adduct of [(p-AA-CH$-\mathrm{HP})_{1} / \mathrm{HB}_{5}+$ $\mathrm{Na}]^{+}$(co)oligoester at $\mathrm{m} / \mathrm{z} 841$ terminated by p-anisic acid and carboxyl end groups containing one p-AA- $\mathrm{CH}_{2}-\mathrm{HP}$ comonomer unit.

Scheme 3. Theoretical fragmentation pathway of the sodium adduct of [p-AA-(p-AA- $\mathrm{CH}_{2}-$ $\left.\mathrm{HP})_{2} / \mathrm{HB}_{2}+\mathrm{Na}\right]^{+}$(co)oligoester at $m / z 819$ terminated by p-anisic acid and carboxyl end groups containing two p-AA- $\mathrm{CH}_{2}-\mathrm{HP}$ comonomer units. 